\title{
Is molecular biology yet a science?
}

The great successes of the past few years suggest that living processes consist of well-ordered events executed under strict control, but a few numbers would give a different and more fuzzy impression.

NoBODY can now shrug off the triumphant pervasiveness of molecular biology, which touches everything in the life sciences and which also affects the character of research. There is now an army of people called molecular biologists whose published papers are innocent of references to whole plants and animals and which may have little to say about their physiology either. For these people, experimental data may consist largely of what are called, in the trade, 'gels' - perhaps simply strips of filter paper allowing the comparison of the molecular weights of large molecules with those of similar materials by the relative rate at which they move under the influence of an electric field. Such techniques are deliciously simple, at least in skilled hands. It is marvellous that so much continues to be learned from them.

The other side of this coin is that molecular biology seems well on the way to becoming a largely qualitative science. The notion that science hangs on measurement seems to have been diminished (although, to be fair, electrophoresis is a measurement of a kind). For some purposes, that may not matter. If the objective is simply to tell the difference between the normal version of a gene and that which appears to be responsible for an inherited disease, then it may suffice to isolate fragments of each (by electrophoresis, for example) and then to determine their nucleotide sequences.

The worry is that, in the long run, strictly qualitative information such as that will not suffice. If some intermediate goal in biology is an understanding of the functioning of an entire cell, it is unthinkable that it will be attainable without quantitative information about the abundance of the component molecular species. But it is also possible that many subcellular processes on which attention is now concentrated will not be intelligible without new quantitative information.

To what extent, for example, is the timing of the transcription of a particular gene determined by the concentration of the particular protein molecule (the 'transcription factor') that must bind to all-purpose RNA polymerase before transcription can begin? And exactly how efficient are the processes by which membrane and other proteins migrate to the places in a cell where they 'belong'? If, in the development of an organism, the emergence of different parts is determined by the diffusion of molecular morphogens, what concentrations of them suffice for normal development?

In the biochemical economy of a cell, such questions are far from irrelevant. If, for example, the transcription of a gene requires a protein specific to that gene, and then the formation of a complex between that protein and a suitably placed molecule of RNA polymerase, it is improbable that the pre-synthesis of a single molecular copy of the initiation factor will suffice. But the synthesis of more than a single molecule may be, strictly speaking, a waste of free energy. Is it not crucial to the understanding of the cell to know how great is the implied inefficiency?

In those bacterial systems in which cells are spontaneously transformed from one form into another, it seems that the accumulation of the transcription factor necessary to transcribe the genes active in one stage may also be the trigger for the transition to the next stage. The relationships between the regulatory processes concerned are intricate and, in their own way, elegant. But those concerned with them seem generally to be more excited by the intricacies than with the definition of what concentration of this or that element of the regulatory apparatus is needed for orderly development.

That is not only a misfortune in its own right, but part of the reason why those seeking explanations of, say, the onset of cell division persistently look for qualitative triggers when quantitative triggers - such as the accumulation of unwanted products of previous stages may be a large part of the answer. More generally, a greater concern for the quantitative features of gene control might throw light on phenomena such as that in which children produce both fetal and adult haemoglobin for some months after birth, which could well be relevant to the understanding of maturation.

Curiously enough, even when molecular biology derives from fields in which there is a strong tradition of measurement, neurophysiology for example, attempts to make arguments quantitative appear to be neglected. To be sure, it is possible, using standard thermodynamic arguments, to relate the membrane potential of a neuron to the ratio of the concentration of potassium and other ions inside and outside the cell, but what does that imply for the rate at which such a cell must generate ATP so as to maintain the polarized state, let alone to recovering from depolarization? The sums can obviously be done, but are their implications explored?

Even the textbooks are not enlightening. For instance, Molecular Biology of the Cell (Alberts et al.) and Molecular Cell Biology (Darnell, Lodish and Baltimore) have impeccable accounts of how mitochondria convert the energy of the respiratory cycle into ATP by maintaining a gradient of hydrogen ions across the inner membrane, but neither goes so far as to describe even back-of-the-envelope calculations to suggest what quantities and concentration may be involved.

There are two important consequences of the neglect of quantitative considerations in molecular biology, one of which is psychological: so long as people search for (and continue to find) qualitative explanations for phenomena in cell biology, they will give credence to the view that the average cell is just a bag filled with molecular switches that exist to be turned off and on as the appropriate molecular actuators make their appearance in some predetermined sequence. That helps to give the impression that the reductionist agenda would make the description of living things neatly cut and dried, which could not be further from the truth.

More seriously, the neglect of quantitative considerations may well be a recipe for overlooking problems inherently of great importance. Enquiries as different as the search for the origin of life and the understanding of the efficacy of the secretory mechanism in cells require not just the knowledge of what molecular processes are energetically possible but also some understanding of the concentrations in which participating chemicals may realistically be found. And there is every likelihood that, then, the dynamics of these processes would significantly limit the range of what is otherwise possible.

So should molecular biologists mend their ways, resurrecting the Law of Mass Action (now conspicuous by its absence from what they publish)? That would be to ask too much when there is so much to be learned from qualitative relationships. But it would be a worthwhile precaution against the quantitative days that lie ahead that people should make sure that published data are capable of quantitative interpretation by those who have the zeal for that. As things are, that is far from true.

John Maddox 